\title{
DIAGNOSE NUTRICIONAL DO CAFEEIRO PELO DRIS VARIANDO-SE A CONSTANTE DE SENSIBILIDADE DOS NUTRIENTES DE ACORDO COM A INTENSIDADE E FREQÜÊNCIA DE RESPOSTA NA PRODUÇÃO ${ }^{(1)}$
}

\author{
ONDINO CLEANTE BATAGLIA ${ }^{(2,4)}$; JOSÉ ANTONIO QUAGGIO(2); \\ WAGNER RODRIGUES DOS SANTOS(3); MÔNICA FERREIRA DE ABREU(2)
}

\begin{abstract}
RESUMO
O objetivo deste trabalho foi testar a hipótese de usar escores diferenciados para nutrientes irresponsivos (I), medianamente responsivos (M) e responsivos (R), como forma de reduzir os efeitos da interdependência no cálculo dos índices do DRIS (Sistema Integrado de Diagnose e Recomendação) e seus efeitos na diagnose nutricional do cafeeiro. O trabalho foi desenvolvido usando os resultados do monitoramento nutricional, pela análise foliar, de talhões de cafeeiros em empresas produtoras situadas nos municípios de Matão e Franca (SP). Os dados foram coletados durante os anos de 1999 a 2001 . Os cálculos dos índices do DRIS foram feitos usando-se a fórmula proposta em 1981 por Jones, em sua forma original com uma constante de sensibilidade $\mathrm{k}=30$ para todas as relações entre nutrientes, ou atribuindo diferentes valores para a constante de sensibilidade em função da resposta do cafeeiro aos nutrientes. Os resultados, quando avaliados pela relação entre o índice de balanço nutricional médio (IBNm) e a produtividade, mostraram que a atribuição de escores diferenciados na ordem $\mathrm{I}<\mathrm{M}<\mathrm{R}$ mostrou maior eficiência quando comparada ao método tradicional de cálculo dos índices DRIS. A melhor relação foi 1-6-10 e a pior, 1-10-1. Na análise comparativa com os diagnósticos obtidos pelo critério de faixas de suficiência (CFS) o uso de escores diferenciados para o cálculo do DRIS mostrou desempenho ligeiramente superior para as melhores relações (I-M-R) quando se compararam as melhores e as piores relações, porém os ganhos em relação ao procedimento tradicional de cálculo, cujo peso relativo de todos os nutrientes é o mesmo, foram pequenos, indicando a necessidade de ajustes locais para a diagnose nutricional do cafeeiro pelo critério de faixas de suficiência.
\end{abstract}

Palavras-chave: café, DRIS, diagnose foliar, estado nutricional.

\section{ABSTRACT \\ NUTRIENT DIAGNOSIS OF COFFEE-TREE BY DRIS USING DIFFERENT SENSITIVITY CONSTANTS ACCORDING TO THE NUTRIENT YIELD RESPONSE}

The objective of this study was to test the use of standard scores to reduce the dependence among nutrients on DRIS indices. Different scores were attributed to nutrients with common (R), intermediate (M) and rare (I) response to coffee crop. The study was conducted using data of leaf analysis from plantation farms at Matão and Franca, State of São Paulo, Brazil. Leaves were taken from bearing branches during the summer, from 1999 to 2001. DRIS indices were calculated using the original formula defined in 1981 by JONES with a sensitivity constant $\mathrm{k}=30$ for all nutrient relations. The tested method attributed different

( $\left.{ }^{1}\right)$ Trabalho financiado pelo Consórcio Brasileiro de Pesquisa e Desenvolvimento do Café (CBP\&D Café). Recebido para publicação em 7 de agosto de 2002 e aceito em 3 de março de 2004.

$\left(^{2}\right)$ Centro de Pesquisa e Desenvolvimento de Solos e Recursos Ambientais, Instituto Agronômico (IAC), Caixa Postal 28, 130001-970 Campinas (SP). E-mail: ondino@iac.sp.gov.br

$\left(^{3}\right)$ Engenheiro Agrícola, MSc., TASQA Serviços Analíticos Ltda., Avenida José Paulino, 1370, 13140-000 Paulínia (SP).

$\left({ }^{4}\right)$ Bolsista do CNPq. 
values for the constant $k$, depending on the type of response of coffee-tree to each nutrient. When the methods were evaluated by comparison of the relations between the nutrient balance index (NBI) and yield, the use of scores in the order $\mathrm{I}<\mathrm{M}<\mathrm{R}$ showed to be more efficient then the usual method using the same score for all nutrients. The methods had about the same efficiency when evaluated by comparing the leaf nutrient diagnosis by DRIS with the sufficiency range method.

Key words: coffee, DRIS, leaf analysis, nutrient status.

\section{INTRODUÇÃO}

O monitoramento nutricional de cafezais mediante análise química das folhas vem-se transformando em prática essencial para recomendações de adubações mais equilibradas e economicamente mais ajustadas. Assim, RAIJ et al. (1997) e GUIMARÃEs et al. (1999) estabelecem as recomendações de adubação nitrogenada em função da produtividade esperada e do teor de $\mathrm{N}$ nas folhas do cafeeiro. Outros sistemas, como o Programa de Adubação Modular (Romero e Romero, 2000), fazem uso da análise foliar para ajuste de recomendação de todos os nutrientes. Esses critérios para avaliação do estado nutricional do cafeeiro baseiam-se nos conceitos de nível crítico e de faixas de concentração, caracterizados pela independência entre nutrientes. O teor de um nutriente não afeta a classificação dos demais. Por outro lado, possui a grave limitação de não identificar quais nutrientes são mais limitantes (TRANI et al., 1983; RAIJ et al., 1997; MARTINEZ et al., 1999; Martinez et al., 2000; Romero e Romero, 2000).

Algumas das limitações dos critérios acima descritos para avaliação do estado nutricional do cafeeiro foram superadas pelo sistema integrado de diagnose e recomendação (DRIS), desenvolvido originalmente por BEAUfILS (1973). O sistema permite o cálculo de índices para cada nutriente, usando as relações dele com os demais e comparando-as com uma população de referência. Esses índices apresentam a vantagem de possibilitar um ordenamento de nutrientes, desde os mais limitantes até os excessivos. Todavia, se por um lado a interdependência permite uma classificação e ordenamento de nutrientes, também pode se transformar em grande desvantagem traduzida em falsos diagnósticos, em função das distorções provocadas por fatores não controlados (Baldock e Schulte, 1989; Beverly, 1993).

Para qualquer fórmula de cálculo dos índices DRIS (Beaufils, 1973; Jones, 1981; Elwali e Gascho, 1984), os efeitos da interdependência podem ser agravados quando ocorrem contaminações das amostras por resíduos de nutrientes aplicados via foliar, principalmente em plantas perenes como o café (BATAGLIA et al., 2000). Além dessas contaminações, alguns nutrientes cuja ocorrência nas plantas é muito variável acabam acarretando prejuízos para outros de composição mais estável. Nutrientes como ferro e manganês, que mostram grande variabilidade de concentrações, principalmente devido às condições edafoclimáticas, são alguns exemplos preocupantes.

Algumas estratégias têm sido testadas para minimizar esses desvios causados pela interdependência no cálculo dos índices. BeVERLY (1987) fez uso de dados com transformação logarítmica visando atenuar grandes variações de concentração de alguns nutrientes, entretanto, o procedimento não mostrou grande eficiência. BALDOck e Schulte (1996) propuseram um sistema com base em escores padronizados visando associar o DRIS com o critério de faixa de suficiência e assim eliminar os freqüentes erros de diagnóstico observados para a cultura do milho. BATAGLIA et al., (2000) sugeriram a restrição de uso de nutrientes em nível de contaminação por resíduos como forma de evitar erros no cálculo dos índices do DRIS de nutrientes não contaminados.

O objetivo deste trabalho foi testar, como forma de reduzir os efeitos da interdependência no cálculo dos índices do DRIS, a hipótese de se trabalhar com valores diferenciados para a constante $\mathbf{k}$ em função da intensidade de resposta de cada nutriente em relação à produtividade do cafeeiro. A proposta, apesar de diferenciada, segue alguns dos princípios estabelecidos por BALDOCK e SCHULTE (1996) para estabelecer o PASS (análise de plantas com escores padronizados), usado com sucesso para milho.

\section{MATERIAL E MÉTODOS}

Os dados analisados neste trabalho foram obtidos de 1999 a 2001, em empresas produtoras de café situadas nos municípios de Matão e Franca, no Estado de São Paulo. Nessas fazendas, os cafezais foram mapeados em talhões, e em cada um foram feitas amostragens de folhas e anotados os dados de produtividade por ano.

A amostragem de folhas foi feita no verão, coletando-se a terceira folha de ramos frutíferos na altura média das plantas em cerca de 50 plantas por talhão, conforme recomendações de RAIJ et al. (1997). 
As amostras, após lavagem, secagem a $60{ }^{\circ} \mathrm{C}$ e moagem, foram submetidas à análise de macro e micronutrientes usando os métodos descritos por BATAGLiA et al. (1983), com as determinações feitas por espectrometria de emissão atômica de plasma induzido (ICP-AES).

A população usada como referência para os cálculos do DRIS foi estabelecida de acordo com os critérios de BEAufILS (1973), considerando-se apenas amostras provenientes de talhões de plantas com produtividade na faixa correspondente à média mais e menos um desvio-padrão da população original e nutricionalmente equilibradas (BATAGLIA et al., 2001). As características da população de referência são apresentadas na tabela 1 para as de relações entre nutrientes.

Tabela 1. Valores das relações entre nutrientes na população de referência para cafeeiros arábica

\begin{tabular}{|c|c|c|c|c|c|}
\hline Relação & Média & Desvio-padrão & Relação & Média & Desvio-padrão \\
\hline $\mathrm{N} / \mathrm{P}$ & 20,2662 & 3,3519 & $\mathrm{Ca} / \mathrm{Mg}$ & 3,4464 & 0,5202 \\
\hline $\mathrm{N} / \mathrm{K}$ & 1,4087 & 0,2142 & $\mathrm{Ca} / \mathrm{S}$ & 5,9810 & 1,0725 \\
\hline $\mathrm{N} / \mathrm{Ca}$ & 2,4586 & 0,3419 & $\mathrm{Ca} / \mathrm{B}$ & 0,2396 & 0,0567 \\
\hline $\mathrm{N} / \mathrm{Mg}$ & 8,3985 & 1,2466 & $\mathrm{Ca} / \mathrm{Cu}$ & 0,9768 & 0,2346 \\
\hline $\mathrm{N} / \mathrm{S}$ & 14,3334 & 2,4925 & $\mathrm{Ca} / \mathrm{Fe}$ & 0,1398 & 0,0328 \\
\hline N/B & 0,5864 & 0,1421 & $\mathrm{Ca} / \mathrm{Mn}$ & 0,1067 & 0,0362 \\
\hline $\mathrm{N} / \mathrm{Cu}$ & 2,4401 & 0,5667 & $\mathrm{Ca} / \mathrm{Zn}$ & 1,2686 & 0,4709 \\
\hline $\mathrm{N} / \mathrm{Fe}$ & 0,3402 & 0,0797 & $\mathrm{Mg} / \mathrm{S}$ & 1,7484 & 0,3083 \\
\hline N/Mn & 0,2587 & 0,0826 & $\mathrm{Mg} / \mathrm{B}$ & 0,0699 & 0,0146 \\
\hline $\mathrm{N} / \mathrm{Zn}$ & 3,0223 & 1,1305 & $\mathrm{Mg} / \mathrm{Cu}$ & 0,2755 & 0,0664 \\
\hline $\mathrm{P} / \mathrm{K}$ & 0,0711 & 0,0125 & $\mathrm{Mg} / \mathrm{Fe}$ & 0,0393 & 0,0111 \\
\hline $\mathrm{P} / \mathrm{Ca}$ & 0,1233 & 0,0199 & $\mathrm{Mg} / \mathrm{Mn}$ & 0,0305 & 0,0098 \\
\hline $\mathrm{P} / \mathrm{Mg}$ & 0,4280 & 0,0730 & $\mathrm{Mg} / \mathrm{Zn}$ & 0,3666 & 0,1151 \\
\hline $\mathrm{P} / \mathrm{S}$ & 0,7323 & 0,1179 & S/B & 0,0397 & 0,0089 \\
\hline $\mathrm{P} / \mathrm{B}$ & 0,0292 & 0,0076 & $\mathrm{~S} / \mathrm{Cu}$ & 0,1561 & 0,0341 \\
\hline $\mathrm{P} / \mathrm{Cu}$ & 0,1222 & 0,0340 & $\mathrm{~S} / \mathrm{Fe}$ & 0,0228 & 0,0056 \\
\hline $\mathrm{P} / \mathrm{Fe}$ & 0,0169 & 0,0037 & $\mathrm{~S} / \mathrm{Mn}$ & 0,0178 & 0,0057 \\
\hline $\mathrm{P} / \mathrm{Mn}$ & 0,0131 & 0,0045 & $\mathrm{~S} / \mathrm{Zn}$ & 0,1983 & 0,0640 \\
\hline $\mathrm{P} / \mathrm{Zn}$ & 0,1503 & 0,0571 & $\mathrm{~B} / \mathrm{Cu}$ & 3,8522 & 1,0721 \\
\hline $\mathrm{K} / \mathrm{Ca}$ & 1,7709 & 0,3320 & $\mathrm{~B} / \mathrm{Fe}$ & 0,6068 & 0,1861 \\
\hline $\mathrm{K} / \mathrm{Mg}$ & 6,0288 & 0,9876 & $\mathrm{~B} / \mathrm{Mn}$ & 0,4350 & 0,1489 \\
\hline $\mathrm{K} / \mathrm{S}$ & 10,4020 & 1,6985 & $\mathrm{~B} / \mathrm{Zn}$ & 5,1375 & 1,7995 \\
\hline $\mathrm{K} / \mathrm{B}$ & 0,4241 & 0,0910 & $\mathrm{Cu} / \mathrm{Fe}$ & 0,1439 & 0,0437 \\
\hline $\mathrm{K} / \mathrm{Cu}$ & 1,6844 & 0,4735 & $\mathrm{Cu} / \mathrm{Mn}$ & 0,1001 & 0,0384 \\
\hline $\mathrm{K} / \mathrm{Fe}$ & 0,2416 & 0,0646 & $\mathrm{Cu} / \mathrm{Zn}$ & 1,5445 & 0,4782 \\
\hline $\mathrm{K} / \mathrm{Mn}$ & 0,1868 & 0,0608 & $\mathrm{Fe} / \mathrm{Mn}$ & 0,8251 & 0,3082 \\
\hline \multirow[t]{2}{*}{$\mathrm{K} / \mathrm{Zn}$} & 2,0997 & 0,7350 & $\mathrm{Fe} / \mathrm{Zn}$ & 9,6458 & 4,1574 \\
\hline & & & $\mathrm{Mn} / \mathrm{Zn}$ & 13,3186 & 5,9303 \\
\hline
\end{tabular}


Para o cálculo dos índices do DRIS, aplicouse a fórmula geral proposta por BEAUfILS (1973), sendo para um nutriente $Y$ :

$$
I Y=\frac{\sum_{i=1}^{n} f\left(Y / X_{i}\right)-\sum_{j=1}^{m} f\left(X_{j} / Y\right)}{n+m}
$$

Os valores das funções intermediárias $\mathrm{f}(\mathrm{Y} / \mathrm{X})$ foram obtidos pela fórmula de Jones (1981). Para a relação $\mathrm{N} / \mathrm{P}$, por exemplo, a função intermediária $\mathrm{f}(\mathrm{N} /$ P) foi obtida como segue:

$$
\begin{aligned}
& f(N / P)=\left[(N / P)_{a}-(N / P)_{p}\right] k / s \\
& \text { em que: }
\end{aligned}
$$

$(\mathrm{N} / \mathrm{P})_{\mathrm{a}}=$ relação na amostra; $(\mathrm{N} / \mathrm{P})_{\mathrm{p}}=$ relação na população de referência; $\mathrm{k}=$ constante de sensibilidade; s = desvio-padrão da relação na população de referência.
O índice de balanço nutricional médio (IBNm) para cada amostra foi calculado pela média dos valores absolutos dos índices DRIS de cada nutriente, dividido pelo número de nutrientes envolvidos.

$$
\mathrm{IBNm}=(|\mathrm{IY} 1|+|\mathrm{IY} 2|+\ldots+|\mathrm{IY} \mathrm{n}|) / \mathrm{n}
$$

Os testes de escores diferenciados dos nutrientes, de acordo com o efeito que cada um deles exerce na produtividade do cafeeiro, foram realizados atribuindo-se valores diferenciados aos nutrientes responsivos (R) incluindo-se nesta categoria $N, K, S$, $\mathrm{B}$ e $\mathrm{Zn}$, nutrientes medianamente responsivos (M): $\mathrm{P}$, $\mathrm{Ca}, \mathrm{Mg}$ e $\mathrm{Mn}$ e pouco responsivos (I): $\mathrm{Cu}$ e Fe.

Para testar esses escores diferenciados, os talhões originais usados para o estabelecimento da população de referência deste trabalho, foram agrupados em treze classes de produtividade com os

\begin{tabular}{|c|c|c|c|c|c|c|c|c|c|c|c|}
\hline $\mathrm{N}$ & $\mathrm{P}$ & $\mathrm{K}$ & $\mathrm{Ca}$ & $\mathrm{Mg}$ & $\mathrm{S}$ & B & $\mathrm{Cu}$ & $\mathrm{Fe}$ & $\mathrm{Mn}$ & $\mathrm{Zn}$ & Produção \\
\hline \multicolumn{6}{|c|}{$\longrightarrow$ g.kg $\mathrm{kg}^{-1}$} & \multicolumn{5}{|c|}{$-\mathrm{mg} \cdot \mathrm{kg}^{-1}$} & kg.ha ${ }^{-1}$ \\
\hline 32,7 & 1,7 & 23,5 & 12,8 & 3,8 & 2,2 & 51,8 & 14,5 & 115,0 & 99,9 & 10,9 & 3.114 \\
\hline 31,8 & 1,7 & 24,0 & 13,5 & 3,8 & 2,3 & 58,2 & 16,4 & 98,2 & 133,5 & 15,2 & 2.853 \\
\hline 31,9 & 1,6 & 22,6 & 12,9 & 3,9 & 2,2 & 47,5 & 13,9 & 104,4 & 122,0 & 12,5 & 2.608 \\
\hline 32,4 & 1,6 & 23,3 & 13,1 & 3,9 & 2,1 & 57,9 & 14,8 & 113,0 & 148,7 & 14,6 & 2.350 \\
\hline 32,2 & 1,6 & 21,2 & 12,7 & 3,9 & 2,2 & 52,6 & 14,8 & 100,4 & 186,3 & 14,0 & 2.101 \\
\hline 32,0 & 1,6 & 22,8 & 13,4 & 4,0 & 2,3 & 54,5 & 14,6 & 103,2 & 149,4 & 14,2 & 1.852 \\
\hline 32,7 & 1,7 & 23,3 & 12,8 & 4,0 & 2,2 & 59,1 & 14,7 & 107,1 & 175,5 & 15,1 & 1.591 \\
\hline 31,5 & 1,9 & 24,5 & 13,5 & 4,1 & 2,5 & 55,7 & 14,6 & 106,0 & 164,5 & 16,1 & 1.355 \\
\hline 31,6 & 1,5 & 23,1 & 12,8 & 4,2 & 2,1 & 55,0 & 17,3 & 96,4 & 190,0 & 14,0 & 1.125 \\
\hline 32,0 & 1,5 & 24,0 & 11,5 & 3,9 & 2,1 & 49,1 & 18,6 & 91,9 & 160,7 & 18,8 & 848 \\
\hline 32,9 & 1,5 & 24,2 & 11,9 & 4,2 & 2,0 & 57,1 & 10,7 & 77,7 & 151,3 & 17,9 & 600 \\
\hline 31,8 & 1,7 & 26,8 & 12,7 & 4,0 & 2,3 & 60,4 & 14,6 & 85,7 & 124,6 & 16,1 & 349 \\
\hline 32,4 & 1,7 & 28,0 & 12,6 & 3,6 & 2,2 & 53,3 & 14,6 & 119,2 & 156,8 & 16,6 & 155 \\
\hline
\end{tabular}
respectivos teores médios de nutrientes nas folhas, conforme apresentação na tabela 2.

Tabela 2. Teores médios de nutrientes em folhas de cafeeiros com diferentes classes de produtividade

Para o cálculo dos índices, os valores de $\mathbf{k}$ da fórmula foram ajustados em função dos grupos de nutrientes (I, M ou R). Foram simuladas todas as combinações atribuindo-se para os nutrientes irresponsivos ( $\mathrm{Cu}$ e $\mathrm{Fe}$ ) valores de 1 a 5 e para os medianamente responsivos e responsivos de 1 a 10 . Assim, foram simulados cálculos dos índices pelo método tradicional (DRISJ), quando $\mathrm{I}=1, \mathrm{M}=1$ e $\mathrm{R}=1$. Nesse caso, do cálculo tradicional pela fórmula de
JONES (1981), o escore atribuído a todos os nutrientes tem o valor 1 e a constante $\mathrm{k}=30$. As outras combinações I-M-R variaram desde 1-1-2, com $\mathrm{k}=14,35$ até 5-10-10 com $\mathrm{k}=0,44$. Atribuindo-se valores diferenciados para os nutrientes classificados como I, $\mathrm{M}$ ou $\mathrm{R}$, a constante de sensibilidade $\mathbf{k}$ foi ajustada de modo que seu valor ponderado médio ficasse sempre na faixa de 29,5 a 30,5 e, dessa forma, os índices pudessem ser comparados em uma mesma ordem de grandeza. 
A interpretação do estado nutricional do cafeeiro pelo DRIS para separação de diagnósticos (baixo, adequado, alto) foi feita utilizando-se o conceito de potencial de resposta proposto por WADT (1996), sendo considerados adequados, nutrientes cujos índices do DRIS, em valores absolutos, não excedessem o valor do IBNm abaixo e acima de zero. Nutrientes com índices negativos em valores absolutos maiores do que o IBNm foram considerados baixos. Índices positivos com valor absoluto maior do que o IBNm foram considerados altos para fins de diagnóstico.

Os critérios para avaliação da eficiência dos escores diferenciados foram indicados com base na relação IBNm x produtividade, considerando-se para isso as treze classes dentro da população de referência. Usou-se também a comparação do diagnóstico pelo DRIS com as diferentes relações com o CFS. Nesse caso, procederam-se comparações dos diagnósticos pelo DRIS e CFS, atribuindo-se valor 1 para coincidência de diagnóstico e valor 0 para a não-coincidência. Esse procedimento foi feito com as análises dos talhões comerciais de café de Matão e de Franca em 1999, 2000 e 2001. Foram calculadas as porcentagens de coincidência de diagnóstico. A análise estatística foi feita aplicando-se a transformação $(x+0,5)^{1 / 2}$. As médias foram comparadas pelo teste t a $5 \%$.

\section{RESULTADOS E DISCUSSÃO}

Para os estudos propostos neste trabalho, as amostras de folhas de uma população abrangente de cafeeiros arábica foram grupadas em treze classes com médias de produtividade entre 155 e 3.114 kg.há ${ }^{-1}$ de café beneficiado (Tabela 2).

Como se tratam de médias, há certa uniformização dos valores. Dessa forma, apesar das diferenças marcantes de produtividade, as maiorias absolutas são consideradas como teores adequados quando se usa o CFS para diagnóstico nutricional. Já na tabela 3, os índices do DRIS começam evidenciar diferenças não perceptíveis pelo critério de faixas.

Alguns nutrientes anteriormente classificados como adequados (Ca) ou altos (S) mostraram índices negativos, o que determina um diagnóstico diferenciado. Pode-se verificar também nessa tabela a relação inversa entre o IBNm e a produtividade, fato bastante documentado na literatura do DRIS. Observe-se que para a combinação $\mathrm{I}=1, \mathrm{M}=6$ e $\mathrm{R}=10$ (Tabela 4), há melhor evidência dos diagnósticos identificando diversos distúrbios nutricionais não detectados pelo CFS e pelo cálculo tradicional do DRIS quando todos os nutrientes tem o mesmo escore 1.

Tabela 3. Índices DRIS calculados para os dados da tabela 2 usando se a fórmula tradicional de Jones com escores para I-M-R 1-1-1 e $\mathrm{k}=30$

\begin{tabular}{|c|c|c|c|c|c|c|c|c|c|c|c|c|}
\hline $\mathrm{N}$ & $\mathrm{P}$ & $\mathrm{K}$ & $\mathrm{Ca}$ & $\mathrm{Mg}$ & $S$ & B & $\mathrm{Cu}$ & $\mathrm{Fe}$ & $\mathrm{Mn}$ & $\mathrm{Zn}$ & IBNm & Produção \\
\hline & & & & & & & & & & & & kg.ha ${ }^{-1}$ \\
\hline 2 & 7 & 0 & -7 & -1 & -1 & -6 & 4 & 17 & -20 & 5 & 6 & 3.114 \\
\hline-11 & 0 & -4 & -5 & -8 & -1 & 1 & 10 & -6 & -2 & 25 & 7 & 2.853 \\
\hline 0 & -7 & -4 & -3 & 4 & 5 & -16 & -1 & 8 & -3 & 16 & 6 & 2.608 \\
\hline-5 & -6 & -6 & -8 & -1 & -15 & 2 & 0 & 11 & 6 & 23 & 8 & 2.350 \\
\hline-2 & -10 & -18 & -10 & 3 & -1 & -6 & 2 & 1 & 19 & 21 & 9 & 2.101 \\
\hline-7 & -12 & -9 & -4 & 2 & 3 & -4 & -1 & 2 & 7 & 22 & 7 & 1.852 \\
\hline-7 & -5 & -9 & -14 & 1 & -5 & 2 & -3 & 3 & 14 & 24 & 8 & 1.591 \\
\hline-18 & 9 & -7 & -11 & 0 & 7 & -8 & -7 & -1 & 9 & 26 & 9 & 1.355 \\
\hline-9 & -16 & -7 & -11 & 9 & -10 & -4 & 16 & -6 & 19 & 19 & 11 & 1.125 \\
\hline-2 & -18 & 3 & -24 & 0 & -10 & -15 & 26 & -9 & 10 & 37 & 14 & 848 \\
\hline 9 & -14 & 9 & -13 & 21 & -11 & 9 & -32 & -24 & 10 & 37 & 17 & 600 \\
\hline-9 & -1 & 16 & -13 & 0 & -1 & 7 & -1 & -20 & -6 & 29 & 9 & 349 \\
\hline-8 & -7 & 22 & -17 & -21 & -7 & -10 & -3 & 14 & 8 & 29 & 13 & 155 \\
\hline
\end{tabular}


Tabela 4. Índices DRIS calculados para os teores foliares das amostras da tabela 2 usando os escores para IM-R de 1-6-10, k=0,64 combinação que mostrou melhor correlação entre IBNm e produção

\begin{tabular}{|c|c|c|c|c|c|c|c|c|c|c|c|c|c|}
\hline Classe & $\mathrm{N}$ & $\mathrm{P}$ & K & $\mathrm{Ca}$ & $\mathrm{Mg}$ & $\mathrm{S}$ & B & $\mathrm{Cu}$ & $\mathrm{Fe}$ & $\mathrm{Mn}$ & $\mathrm{Zn}$ & IBNm & Produção \\
\hline & & & & & & & & & & & & & kg.ha ${ }^{-1}$ \\
\hline 1 & 7 & 8 & 4 & -5 & 0 & 1 & -7 & 1 & 3 & -16 & 6 & 5 & 3.114 \\
\hline 2 & -16 & 0 & -7 & -4 & -8 & -1 & 2 & 1 & -1 & -1 & 34 & 7 & 2.853 \\
\hline 3 & 2 & -6 & -5 & -2 & 4 & 9 & -24 & 0 & 1 & -2 & 22 & 7 & 2.608 \\
\hline 4 & -5 & -3 & -8 & -6 & 0 & -22 & 4 & 0 & 2 & 6 & 32 & 8 & 2.350 \\
\hline 5 & 0 & -8 & -26 & -8 & 3 & -1 & -9 & 0 & 0 & 18 & 29 & 9 & 2.101 \\
\hline 6 & -9 & -11 & -14 & -3 & 2 & 5 & -6 & 0 & 0 & 6 & 30 & 8 & 1.852 \\
\hline 7 & -9 & -4 & -14 & -12 & 0 & -8 & 3 & -1 & 0 & 13 & 33 & 9 & 1.591 \\
\hline 8 & -27 & 8 & -11 & -9 & -1 & 10 & -13 & -1 & 0 & 8 & 36 & 11 & 1.355 \\
\hline 9 & -10 & -13 & -9 & -8 & 9 & -13 & -4 & 2 & -1 & 18 & 28 & 10 & 1.125 \\
\hline 10 & 0 & -16 & 7 & -21 & 0 & -15 & -22 & 4 & -1 & 10 & 54 & 14 & 848 \\
\hline 11 & 3 & -19 & 4 & -19 & 11 & -29 & 2 & -6 & -4 & 7 & 49 & 14 & 600 \\
\hline 12 & -19 & -5 & 18 & -15 & -4 & -8 & 5 & -1 & -3 & -6 & 38 & 11 & 349 \\
\hline 13 & -12 & -6 & 34 & -16 & -21 & -12 & -16 & -1 & 2 & 7 & 41 & 15 & 155 \\
\hline
\end{tabular}

A relação característica do DRIS - IBNm x produtividade - foi usada como ferramenta para se testar a eficiência das combinações de escores atribuídos aos nutrientes classificados como I, M ou R, admitindose que o sinal negativo dos coeficientes de correlação mostrem a relação inversa entre DRIS - IBNm e produtividade, e os valores absolutos maiores indiquem melhor desempenho da combinação de escores atribuídos aos nutrientes com respostas diferenciais na nutrição do cafeeiro. Os dados da tabela 5 mostram os resultados desse teste para combinações de nutrientes responsivos e medianamente responsivos (variação de 1 a 10) fixando-se o escore 1 para os nutrientes irresponsivos. Os dados revelam a existência de uma população de combinações que apresentaram melhores coeficientes de correlação, com valores abaixo de $-0,900$. O maior valor $(\mathrm{r}=-0,923)$ foi obtido com a relação 1-6-10, embora valores muito próximos tenham sido observados para outras combinações. $\mathrm{O}$ pior coeficiente de correlação foi verificado para a combinação 1-10-1 $(r=-0,544)$

A figura 1 mostra a dispersão das relações entre os valores de IBNm e a produtividade para três combinações de escores dos grupos de nutrientes. Os gráficos mostram o melhor ajuste linear entre IBNm e produtividade, em que o melhor ajuste corresponde ao tratamento 1-6-10, com coeficiente de determinação $R^{2}=0,8516$ e o pior, ao tratamento $1-10-1$ com $R^{2}$ $=0,2957$. O tratamento 1-1-1, que corresponde ao método tradicional de cálculo dos índices do DRIS, mostrou um comportamento intermediário, $\mathrm{R}^{2}=$ 0,6143 , evidenciando por essa abordagem ser possível melhorar o diagnóstico nutricional, atribuindo-se diferentes níveis de sensibilidade a nutrientes com maior ou menor resposta na produtividade do cafeeiro.

Conforme foi tratado anteriormente na discussão da tabela 5, há uma série de combinações eficientes, o que pode ser bem visualizada nas superfícies de resposta da figura 2. À medida que se atribuem escores mais elevados (4 ou 5) para os nutrientes irresponsivos, o número de combinações de M e R com elevados coeficientes de correlação, em valor absoluto, é muito reduzido. Observam-se escores em que $\mathrm{I}<\mathrm{M}<\mathrm{R}$ tende a apresentar melhores correlações entre IBNm e produtividade.

Atribuindo-se valor 1 para os nutrientes irresponsivos, nota-se uma grande área de valores de r entre - 0,95 e 0,90 . É possível destacar muitas combinações para M e R. Algumas combinações eficientes, ocupando pontos centrais dessas superfícies, podem ser destacadas como: 1-3-6; 1-4-7; 1-5-8; 1-6-10. Para valores $I=2$, a superfície de valores na mesma faixa de coeficientes de correlação é menor, mas mesmo assim podem-se destacar alguns pontos como 2-3-7; 2-4-8 e 2-5-9. Quando I = 3, a superfície dos coeficientes de correlação, ainda na mesma faixa, é bem restrita, mesmo assim podem-se identificar as combinações 3-4-10 e 3-5-10. Para combinações com I = 4 ou 5, não ocorre nenhuma combinação com valores de $\mathbf{r}$ abaixo de $-0,900$. 

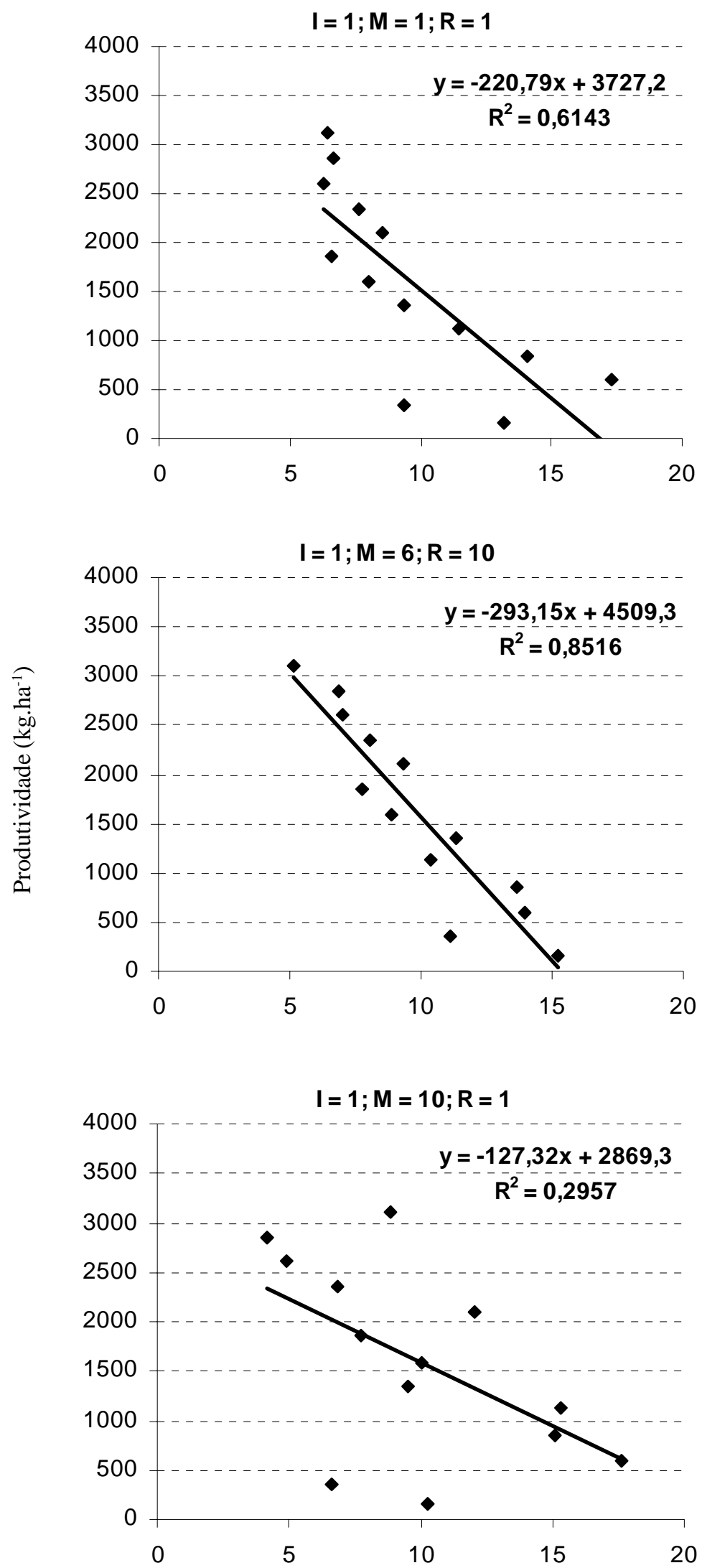

Figura 1. Relação entre os valores de IBNm e a produtividade do cafeeiro obtidas com combinações de escores para nutrientes irresponsivos (I), medianamente responsivos (M) e responsivos (R). 

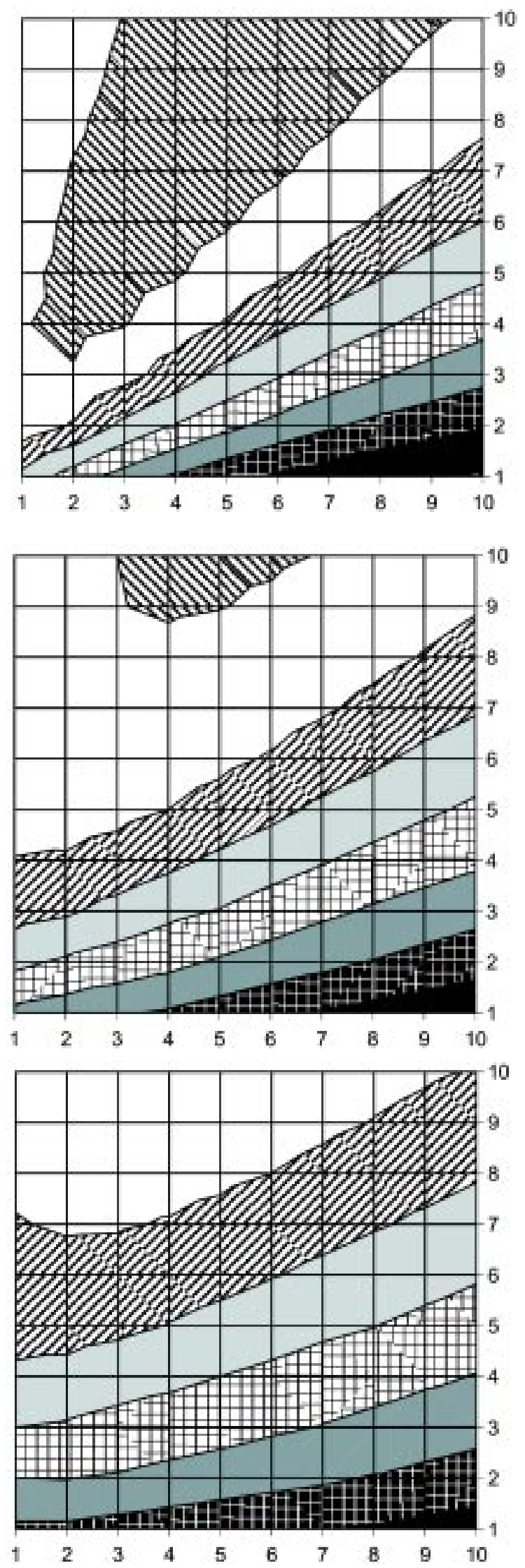
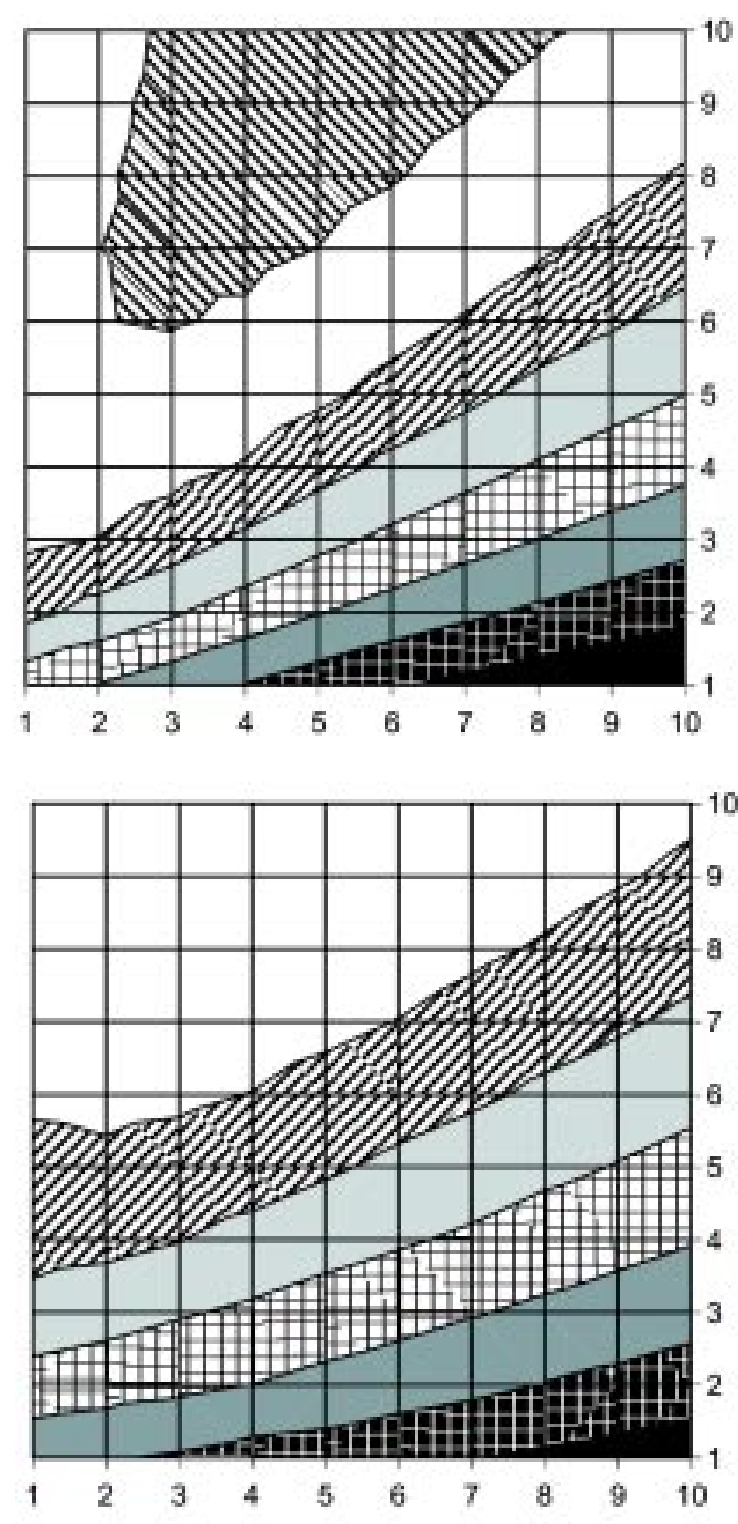

Intervalo de

Coeficientes de Correlação

- $0,60-0,55$

曲 $-0,65-0,60$

$\square-0,70-0,65$

田 $-0,75-0,70$

$\square-0,80-0,75$

प7 $-0,85-0,80$

$\square-0,90-0,85$

N-0,95-0,90

Figura 2. Superfícies de resposta de coeficientes de correlação entre IBNm e produtividade para diferentes combinações de valores atribuídos aos nutrientes classificados como Irresponsivos, Medianamente Responsivos e Responsivos (I,M,R). 
Tabela 5. Coeficientes de correlação entre IBNm e produtividade obtidos usando-se escore I=1 e variando-se M e R de 1 a 10.

\begin{tabular}{|c|c|c|c|c|c|c|c|c|c|c|}
\hline \multirow{2}{*}{$\mathrm{R}$} & \multicolumn{10}{|c|}{$\mathrm{M}$} \\
\hline & 1 & 2 & 3 & 4 & 5 & 6 & 7 & 8 & 9 & 10 \\
\hline 1 & $-0,784$ & $-0,728$ & $-0,680$ & $-0,643$ & $-0,614$ & $-0,591$ & $-0,574$ & $-0,560$ & $-0,551$ & $-0,544$ \\
\hline 2 & $-0,880$ & $-0,846$ & $-0,788$ & $-0,746$ & $-0,713$ & $-0,683$ & $-0,657$ & $-0,635$ & $-0,618$ & $-0,603$ \\
\hline 3 & $-0,899$ & $-0,896$ & $-0,867$ & $-0,826$ & $-0,784$ & $-0,753$ & $-0,727$ & $-0,704$ & $-0,684$ & $-0,665$ \\
\hline 4 & $-0,897$ & $-0,911$ & $-0,902$ & $-0,877$ & $-0,846$ & $-0,813$ & $-0,781$ & $-0,757$ & $-0,735$ & $-0,716$ \\
\hline 5 & $-0,890$ & $-0,912$ & $-0,915$ & $-0,904$ & $-0,883$ & $-0,858$ & $-0,832$ & $-0,805$ & $-0,779$ & $-0,759$ \\
\hline 6 & $-0,880$ & $-0,908$ & $-0,918$ & $-0,916$ & $-0,904$ & $-0,887$ & $-0,866$ & $-0,844$ & $-0,821$ & $-0,798$ \\
\hline 7 & $-0,871$ & $-0,902$ & $-0,916$ & $-0,920$ & $-0,916$ & $-0,904$ & $-0,889$ & $-0,872$ & $-0,853$ & $-0,833$ \\
\hline 8 & $-0,863$ & $-0,895$ & $-0,912$ & $-0,920$ & $-0,921$ & $-0,915$ & $-0,904$ & $-0,891$ & $-0,876$ & $-0,859$ \\
\hline 9 & $-0,857$ & $-0,887$ & $-0,907$ & $-0,918$ & $-0,922$ & $-0,921$ & $-0,914$ & $-0,904$ & $-0,892$ & $-0,879$ \\
\hline 10 & $-0,784$ & $-0,880$ & $-0,901$ & $-0,914$ & $-0,921$ & $-0,923$ & $-0,920$ & $-0,913$ & $-0,904$ & $-0,893$ \\
\hline
\end{tabular}

Tabela 6. Porcentagens de coincidências entre os diagnósticos pelo DRIS com escores diferenciados e pelo CFS em Franca (SP).

\begin{tabular}{|c|c|c|c|c|c|c|c|c|c|c|c|c|c|}
\hline \multirow{2}{*}{ Ano } & \multirow{2}{*}{ Relação } & \multicolumn{12}{|c|}{ Macronutrientes } \\
\hline & & \multicolumn{2}{|c|}{$\mathrm{N}$} & \multicolumn{2}{|c|}{$\mathrm{P}$} & \multicolumn{2}{|c|}{ K } & \multicolumn{2}{|c|}{$\mathrm{Ca}$} & \multicolumn{2}{|c|}{$\mathrm{Mg}$} & \multicolumn{2}{|c|}{$S$} \\
\hline \multirow[t]{3}{*}{1999} & $1-1-1$ & 42 & a & 74 & a & 47 & a & 53 & $a b$ & 47 & a & 53 & a \\
\hline & $1-6-10$ & 42 & a & 68 & a & 63 & a & 58 & $\mathrm{a}$ & 42 & a & 26 & $b$ \\
\hline & $1-10-1$ & 11 & $b$ & 32 & $b$ & 47 & a & 37 & $\mathrm{~b}$ & 42 & a & 26 & $b$ \\
\hline \multirow[t]{3}{*}{2000} & $1-1-1$ & 32 & a & 72 & $\mathrm{~b}$ & 32 & $\mathrm{~b}$ & 56 & a & 36 & a & 28 & $\mathrm{~b}$ \\
\hline & $1-6-10$ & 36 & a & 88 & a & 28 & $b$ & 60 & a & 44 & a & 20 & $b$ \\
\hline & $1-10-1$ & 12 & $b$ & 16 & c & 80 & a & 56 & a & 48 & a & 48 & $\mathrm{a}$ \\
\hline \multirow[t]{3}{*}{2001} & $1-1-1$ & 60 & a & 65 & a & 50 & a & 45 & a & 20 & a & 10 & a \\
\hline & $1-6-10$ & 55 & a & 60 & a & 45 & a & 35 & a & 35 & a & 15 & $\mathrm{a}$ \\
\hline & 1-10-1 & 65 & a & 60 & a & 80 & a & 35 & a & 35 & a & 1 & a \\
\hline
\end{tabular}

Médias seguidas por letras diferentes indicam diferenças estatísticas pelo teste t a $5 \%$.

Tabela 7. Porcentagens de coincidências entre os diagnósticos pelo DRIS com escores diferenciados e pelo CFS em Matão (SP)

\begin{tabular}{|c|c|c|c|c|c|c|c|c|c|c|c|c|c|}
\hline \multirow[t]{2}{*}{ Ano } & \multirow[t]{2}{*}{ Relação } & \multicolumn{12}{|c|}{ Macronutrientes } \\
\hline & & \multicolumn{2}{|c|}{$\mathrm{N}$} & \multicolumn{2}{|c|}{$\mathrm{P}$} & \multicolumn{2}{|c|}{$\mathrm{K}$} & \multicolumn{2}{|c|}{$\mathrm{Ca}$} & \multicolumn{2}{|c|}{$\mathrm{Mg}$} & \multicolumn{2}{|c|}{ S } \\
\hline \multirow[t]{3}{*}{1999} & $1-1-1$ & 45 & $\mathrm{a}$ & 83 & a & 48 & a & 62 & a & 52 & a & 38 & $a b$ \\
\hline & $1-6-10$ & 52 & a & 86 & a & 62 & a & 59 & a & 52 & a & 59 & $\mathrm{a}$ \\
\hline & $1-10-1$ & 28 & a & 17 & $\mathrm{~b}$ & 31 & $\mathrm{~b}$ & 24 & $\mathrm{~b}$ & 48 & a & 21 & $\mathrm{~b}$ \\
\hline \multirow[t]{3}{*}{2000} & $1-1-1$ & 34 & a & 66 & a & 10 & a & 66 & $\mathrm{a}$ & 45 & a & 10 & $\mathrm{a}$ \\
\hline & $1-6-10$ & 48 & a & 72 & a & 17 & a & 59 & $a b$ & 66 & a & 14 & a \\
\hline & $1-10-1$ & 59 & a & 48 & $\mathrm{a}$ & 21 & a & 24 & $\mathrm{~b}$ & 34 & a & 01 & a \\
\hline \multirow[t]{3}{*}{2001} & $1-1-1$ & 14 & a & 14 & $a b$ & 55 & $\mathrm{a}$ & 62 & $\mathrm{a}$ & 38 & a & 21 & a \\
\hline & $1-6-10$ & 28 & a & 7 & $\mathrm{~b}$ & 72 & a & 62 & a & 38 & a & 28 & $\mathrm{a}$ \\
\hline & $1-10-1$ & 24 & $\mathrm{a}$ & 38 & $\mathrm{a}$ & 21 & $b$ & 38 & $\mathrm{a}$ & 17 & $b$ & 14 & $\mathrm{a}$ \\
\hline
\end{tabular}

Médias seguidas por letras diferentes indicam diferenças estatísticas pelo teste t a $5 \%$. 
Os piores resultados foram observados para combinações com valores elevados para os nutrientes e irresponsivos ou medianamente responsivos e valores baixos para os responsivos como, por exemplo, as combinações 1-10-1, 3-10-1 e 5-10-1.

O procedimento tradicionalmente usado no país como método de avaliação nutricional do cafeeiro baseia-se no CFS. Esse método mostrou grande evolução sendo indicado pelos principais manuais de recomendação de adubação para a cultura (Malavolta, 1981; Malavolta, 1993; Raij et al., 1997; Guimarães et al., 1999; Thomaziello et al., 2000). Por essa razão, esse critério foi usado como referência para comparar os procedimentos do DRIS ora em estudo.

Os dados das tabelas 6 e 7 calculados a partir das análises de folhas de cafezais de Franca e Matão mostram resultados pouco concordantes entre os diagnósticos pelo CFS e pelo DRIS. Ao contrário do teste anterior, quando a relação 1-6-10 mostrou-se bastante superior a 1-10-1 na relação IBNm x produtividade; nesse caso, não houve superioridade destacada em relação à proporção de coincidências entre os dois critérios de diagnóstico. Pode-se notar ligeira superioridade da relação 1-6-10 sobre a 1-101 , que são casos extremos. Quando se compara com a relação 1-1-1 que é a forma tradicional de cálculo do DRIS, observa-se que a vantagem dos escores diferenciados é menos marcante. Na prática há certa equivalência entre o procedimento tradicional (relação 1-1-1) e o de escores diferenciados (relação 1-6-10).

Os diagnósticos são bastante discrepantes para enxofre, qualquer que seja a modalidade de cálculo, revelando que provavelmente há necessidade de ajuste das faixas de concentração para diagnose nutricional do cafeeiro pelo CFS.

Os dados do presente trabalho permitiram uma avaliação mais criteriosa do uso de escores diferenciados em função da resposta do cafeeiro aos nutrientes. Mesmo sem ter mostrado uma eficiência comparativa ao CFS, as relações entre o balanço de nutrientes e a produtividade possibilitaram uma seleção bastante adequada das melhores relações de sensibilidade para nutrientes irresponsivos, medianamente responsivos e responsivos. A seleção dessas relações mostrou-se objetiva, sem o empirismo observado em outros trabalhos, (BALDOCK e Schulte, 1996).

Outro aspecto relevante deste trabalho foi o uso da técnica de probabilidade de resposta proposta por WADT (1996) como método de diagnóstico. O método permite a avaliação do estado nutricional de cada amostra, com base no IBNm da própria amostra. Apesar disso, ainda há muito espaço para o desenvolvimento de novos métodos de diagnóstico com base nos índices do DRIS.

Uma das possibilidades é o desenvolvimento de faixas de índices com diagnóstico para teores de nutrientes considerados baixos, adequados e altos, com base na distribuição normal de uma população de índices. Assim, a comparação da amostra seria feita com a população a que ela pertence e não apenas com ela mesma, como ocorre com a avaliação realizada pelo procedimento indicado por WADT (1996). Essa pode ter sido uma das razões da falta de coincidência entre os diagnósticos observados para alguns nutrientes. A outra razão é a necessidade de melhor ajuste nos valores das faixas de concentração de nutrientes usadas pelo CFS. Aparentemente, há necessidade de ajustes locais desses valores.

\section{CONCLUSÕES}

1. As relações entre IBNm e produtividade do cafeeiro foram melhores quando se usaram escores diferenciados para calculo do DRIS para o cafeeiro. Os melhores coeficientes de determinação foram obtidos quando os escores seguiram a ordem para os nutrientes: irresponsivos $<$ medianamente responsivos $<$ responsivos.

2. O uso de escores diferenciados não melhorou a praticidade do diagnóstico nutricional do DRIS em relação ao DRIS tradicional, quando comparados ao critério de faixas de suficiência.

\section{REFERÊNCIAS}

BALDOCK, J.O.; SCHULTE, E.E. The DRIS/Calcium problem. In: FERTILIZE, AGLIME \& PEST MANAGEMENT CONFERENCE, $28^{\text {th }}, 1989$, Madison. Proceedings... Madison: Department of Soil Science, University of Wisconsin, 1989. p.36-43.

BALDOCK, J.O.; SCHULTE, E.E. Plant analysis with standardized scores combines DRIS and sufficiency range approaches for corn. Agronomy Journal, Madison, v.88, p.448456, 1996.

BATAGLIA, O. C.; FURLANI, A.M.C.; TEIXEIRA, J.P.F.; FURLANI, P.R.; GALLO, J.R. Métodos de Análise Química de Plantas. Campinas: Instituto Agronômico, 1983, 48p. (Boletim Técnico, 78)

BATAGLIA, O.C.; SANTOS, W.R.; QUAGGIO, J.A. Efeito de contaminações foliares na diagnose nutricional do cafeeiro pelo DRIS. In: SIMPÓSIO DE PESQUISA DOS CAFÉS DO BRASIL, 1, 2000, Poços de Caldas, MG. Resumos expandidos... Brasília: Embrapa Café e MinasPlan, 2000. v.1, p.1343-1346. 
BATAGLIA, O.C.; SANTOS, W.R.; QUAGGIO, J.A. Reference populations for evaluation of the nutritional status of coffee by DRIS. In: HORST, W.J. et al. (Eds.). Plant nutrition: Food security and sustainability of agro-ecosystems. Dordrecht: Kluwer Academic Publishers, 2001. p.728-728.

BEAUFILS, E.R. Diagnosis and Recommendation Integrated Systems (DRIS) a general scheme for experimentation and calibration based on principles from research in plant nutrition. Pietermaritzburg: University of Natal, South Africa, 1973. 132p. (Soil Science Bulletin, I)

BEVERLY, R. B. Comparison of DRIS and alternative nutrient diagnostic methods for soybean. Journal of Plant Nutrition, New York, v.10, p.901-920, 1987.

BEVERLY, R. B. DRIS diagnoses of soybean nitrogen, phosphorus and potassium are unsatisfactory. Journal of Plant Nutrition, New York, v.16, p.1431-1447, 1993.

ELWALI, A.M.O.; GASCHO, J.G. Soil testing, foliar analysis, and DRIS as guide for sugarcane fertilization. Agronomy Journal, Madison, v.76, p.466-470, 1984.

GUIMARÃES, P.T.G.; GARCIA, A.W.R.; ALVAREZ V., V.H.; PREZOTTI, L.C.; VIANA, A.S.; MIGUEL, A.E.; MALAVOLTA, E.; CORREAA, J.B.; LOPES,A.S.; NOGUEIRA, F.D.; MONTEIRO, A.V.C. Cafeeiro. In: RIBEIRO, A.C.; GUIMARÃES, P.T.G.; ALVAREZ V., V.H. (Eds.). Recomendações para o uso de corretivos e fertilizantes em Minas Gerais - $5^{\text {a }}$ Aproximação. Viçosa: Comissão de Fertilidade do Solo do Estado de Minas Gerais, 1999. p.289-302.

JONES, C.A. Proposed modifications of the diagnosis and recommendation integrated system (DRIS) for interpreting plant analyses. Communications in Soil Science and Plant Analysis, New York, v.12, n.8, p.785-794, 1981.

MALAVOLTA, E. Nutrição mineral e adubação do cafeeiro: passado, presente e perspectivas. In: MALAVOLTA, E.; YAMADA, T.; GUIDOLIN, J.A. (Eds.). Nutrição e adubação do cafeeiro. Piracicaba: Instituto da Potassa e Fosfato, Instituto Internacional da Potassa, 1981. p.138-194.
MALAVOLTA, E. Nutrição mineral e adubação do cafeeiro. São Paulo: Editora Agronômica Ceres, 1993. 210p.

MARTINEZ,H.E.P.; CARVALHO, J.G.;SOUZA, R.B. Diagnose foliar. In: RIBEIRO, A.C.; GUIMARÃES, P.T.G.; ALVAREZ V., V.H. (Eds.). Recomendações para o uso de corretivos e fertilizantes em Minas Gerais - $5^{\mathrm{a}}$ Aproximação. Viçosa: Comissão de Fertilidade do Solo do Estado de Minas Gerais, 1999. p.143-168.

MARTINEZ, H.; SOUZA, R.B.; ALVARES, V.V.H.; MENEZES, J.F.S.; OLIVEIRA, J.A.; ALVARENGA, A.P.; GUIMARÃES, P.T.G. Nutrição mineral, fertilidade do solo e produtividade do cafeeiro nas regiões de Manhuaçu e Patrocínio. Belo Horizonte: EPAMIG, 2000.36p.

RAIJ, B. van; FERNANDES, D.R.; OLIVEIRA, E.G.; MALAVOLTA, E. et al. Café. In: RAIJ, B. van; CANTARELLA, H.; QUAGGIO, J.A.; FURLANI, A.M.C. (Eds.). Recomendações de adubação e calagem para o Estado de São Paulo. 2.ed. ver. atual. Campinas: Instituto Agronômico, 1997. p.97-101. (Boletim Técnico, 100)

ROMERO, J.C.P.; ROMERO, J.P. Café : PAM, Programa de Adubação Modular. São Paulo: Agronômica Ceres, 2000. 74p.

THOMAZIELLO, R.A.; FAZUOLI, L.C.; PEZZOPANE, J.R.M.; FAHL, J.I.; CARELLI, M.L.C. Café arábica: cultura e técnicas de produção. Campinas: Instituto Agronômico, 2000. 82p.

TRANI, P.E.; HIROCE, R.; BATAGLIA, O.C. Análise foliar: amostragem e interpretação. Campinas: Fundação Cargill, 1983. 18p.

WADT, P.G.S. Os métodos da chance matemática e do sistema integrado de diagnose e recomendação (DRIS) na avaliação nutricional de plantios de eucalipto. 1996. 123f. Tese (Doutorado) - Universidade Federal de Viçosa. 